\title{
Name or hum that tune: Feeling of knowing for music
}

\author{
ZEHRA F. PEYNIRCIOǦLU, ALI İ. TEKCAN, JENNIFER L. WAGNER, \\ TERRI L. BAXTER, and STEPHANIE D. SHAFFER \\ American University, Washington, D.C.
}

\begin{abstract}
This is the first reported research that explores the feeling of knowing (FOK) for musical stimuli. Subjects attempted to recall melodies and titles of musical pieces, made FOK ratings when recall failed, and then had a recognition test. With instrumental music (Experiment 1), more titles were recalled when melodies were given as cues than vice versa. With songs whose lyrics were not presented (Experiment 2), however, more melodies were recalled than were titles. For nonrecalled items, although the overall levels of recognition did not differ, FOK ratings were higher for titles than for melodies in Experiment 1, and the opposite pattern occurred in Experiment 2. In both experiments, the FOK ratings predicted melody recognition more accurately than they did title recognition.
\end{abstract}

Sometimes we find ourselves humming or whistling a melody but are unable to recall what it is, even though we believe that we know it. Similarly, when someone asks us whether we know a certain piece, we may find ourselves blocking on the melody, even though we believe that we know it and that we will recognize it if we hear it. Just as with words or with names (see, e.g., Hart, 1965), such feelings of knowing (FOKs) may be accompanied by partial information. We may not be able to remember the title, even though we may remember who the composer is or the occasion on which we heard the melody last. Similarly, in the case of the melody, we may not be able to hum the music, even though we may remember that it was a slow romantic piece or that it was a piece for flute. In this paper, we describe the first empirical research on such FOKs for music.

When people are unable to recall something but predict that they would recognize it among alternatives or that they would recall it later on, they are said to be in an FOK state. Over the last 30 years, much effort has been devoted to research on such states and much has been learned. FOK states can occur in episodic memory tasks, such as one in which a subject tries to remember a response term, when given a stimulus term in a paired-associate learning paradigm (see, e.g., Schacter, 1983), as well as in semantic memory tasks, such as one in which a subject tries to answer a general knowledge question or to recall a word when given its definition (see, e.g., Hart, 1965; Nelson, McSpadden, Fromme, \& Marlatt, 1986). Usually, FOK judgments are used to predict whether sub-

We thank all our friends who helped us collect our musical pieces and Rana Tekcan, who helped also with the recording. Correspondence concerning this article should be addressed to $Z$. Peynircioğlu, Department of Psychology, American University, Washington, DC 20016 (e-mail: peynir@american.edu).

-Accepted by previous associate editor Thomas $O$. Nelson jects will recognize the target from among alternatives (see, e.g., Hart, 1965), recall the target when given various cues (see, e.g., Gruneberg \& Monks, 1974), relearn the target faster in another task (see, e.g., Nelson, Gerler, \& Narens, 1984), or even identify the target better in a perceptual identification task (see, e.g., Nelson et al., 1984).

Although people do, in fact, sometimes feel that they know the answer, even though they cannot remember it at the moment, they are not always accurate. In typical research, accuracy is determined by the match between subjects' predictions of whether they will be able to remember the answer in a future test (e.g., recognition from among alternatives or filling in the missing letters) and their performance on that test. Thus, although people are often accurate in their predictions, sometimes they fail to recognize the answer, even when they had predicted that they would, or they do, in fact, recognize the answer, even when they had predicted that they would not.

In this study, we looked at memory for titles of given melodies and for melodies of given titles. We also looked at FOK states. To date, most research on FOK states has used verbal materials. We were interested in exploring whether people could predict what they knew in music, too, and whether there were any asymmetries between the FOK ratings for titles and those for melodies, as well as between the accuracies of such ratings.

Intuitively, melodies and their titles may be less closely related to one another than materials that have been traditionally used in FOK studies of existing knowledge. For instance, definitions are an inherent aspect of words, in that the two convey the same meaning and are interchangeable. In fact, they are derived from each other and can be seen as facets of the same entity; thus, it is probably not often that a word and its definitions are learned separately. Similarly, general knowledge questions are derived from the answer itself, and it is unlikely that one could learn a question and its answer as separate entities. 
Other materials, such as countries and cities, also appear to be closely related, in that often we learn about them in an associative manner (e.g., the capital of Italy is Rome, or Tampere is in Finland).

Melodies and their titles are in different modalities, however, and are neither interchangeable nor primarily learned in an associative manner. For instance, we may learn, purely at the verbal level - say, for a class on music appreciation - that Mozart has 41 symphonies and that the 41 st one is called Jupiter, without necessarily having an inkling about what the melody is. Or we may hear our brother play Beethoven's violin Romance in F and thus come to learn it, purely at the sound level, without knowing what it is. In addition, the titles of many melodies are quite arbitrary in terms of relatedness. There is no inherent difference, for instance, between a Symphony 1 and a Symphony 2 in terms of their content. Possibly it is this arbitrariness, as well as the relatively evanescent and unassociable nature of the sounds themselves, ${ }^{1}$ that prevents many of us from being able to name musical pieces or to remember the melody from the names without overlearning or without some analytic thinking. With a few exceptions (e.g., The Flight of the Bumblebee by Rimsky-Korsakoff), there is very little in a melody that would help cue its title, and vice versa. In this sense, connections between many melodies and their titles may not quite be similar to those between words and their definitions or to those between general knowledge questions and their answers. Thus, one may expect differences in metamemory for musical pieces and, for present purposes, in FOK ratings and their accuracies for melodies and titles, when given each other as memory cues.

We also explored whether it made a difference if a melody had lyrics. The lyrics of a song and its title are in the same modality (verbal) and often semantically somewhat related (e.g., the lyrics in the song Love Story have to do with love). It has also been shown that lyrics and music in songs are intimately connected. For instance, Serafine, Crowder, and Repp (1984) and Serafine, Davidson, Crowder, and Repp (1986) have shown that recognition memory for a newly learned melody was enhanced when it was presented along with the original lyrics; similarly, recognition memory for lyrics was also enhanced when it was presented along with the original melody. In the Serafine et al., experiments, this integrated memory representation was found in episodic memory tasks. Bartlett and Snelus (1980) showed a similar effect in a semantic memory task in which recalling lyrics was enhanced the most when the melody was given as the cue. Perhaps, in our case, the lyrics, even when they were not mentioned, would play a role in evoking the Gestalt and have a mediating effect between the title and the melody, thus affecting FOK ratings and their accuracy.

Finally, we looked at the relationship between further melody recallability and FOK states for titles. At the end of each session, all the snippets of target melodies were played to the subjects, and their task was to hum the next few bars. A melody was deemed further recallable (in its own modality) for the subject if he/she could continue it correctly. One issue of interest was whether such melodies were given higher FOK ratings for their titles and whether these ratings were more accurate. Another issue that emerged post hoc was how FOK ratings predicted further melody recallability, when such recallability was used as a criterion measure just like recognition.

\section{EXPERIMENT 1}

\section{Method}

Subjects. The subjects were 60 students at American University who received extra credit in psychology courses for their participation. None reported hearing problems. Eighteen had had 5 or more years of formal training in music and 14 listened to more than $20 \mathrm{~h}$ of music every week ( 7 were in both categories).

Materials. Through extensive piloting, 144 snippets of highly familiar instrumental pieces were chosen. ${ }^{2}$ Some of the pieces were chosen from the material sets of other experiments conducted in our laboratory because the subjects had indicated that they were familiar with those pieces. Other pieces were chosen from those piloted with about 20 undergraduate and graduate students who heard many melodies and indicated how familiar each melody was on a scale of 1 to 10 , where 10 corresponded to I know the name, the composer, etc. and 1 to I am sure I have not heard it before. We chose those pieces that received mean ratings of between 3 and 8 . The snippets always comprised a portion of one of the main themes of the piece (usually the first theme) and often lasted about two to four measures, until a motif or half the phrase had been stated or until the piece was unambiguously identifiable (in the case of nonsymmetrical phrases). The average length of time a snippet took was about $9.8 \mathrm{sec}$ (range of 3.8-18.8 sec). Some examples include snippets from the opening theme of the first movement of Beethoven's Moonlight Sonata, the theme music of the television show "Star Trek," the beginning of Schumann's Träumerei (Reverie) from Scenes of Childhood, the beginning of R. Strauss's Also Sprach Zarathustra (also identified as the music from the movie 2001: A Space Odyssey), and the main theme from Gershwin's Rhapsody in Blue (also identified as the music of the United Airlines commercial). We should perhaps note that, in cases in which there could be more than one title, because the interest was in obtaining a correct verbal identification when given a melody or vice versa, we thought that either correct title would be acceptable -akin to, say, accepting either Muhammad Ali or Cassius Clay, in response to his photograph, as correct.

For the forced choice recognition test, the items were paired such that, as much as possible, neither the melody nor the title of the target snippet was obviously different from those of the lures. For instance, from the examples given above, Beethoven's Moonlight Sonata was paired with Beethoven's Pathetique Sonata (second movement) and Beethoven's Appasionata Sonata (first movement); the theme music of "Star Trek" was paired with the theme music of E.T. and that of "Battlestar Galactica"; Schumann's Träumerei was paired with Rachmaninoff's Vocalise and Lizst's Liebestraume; Strauss's Also Sprach Zarathustra was paired with Debussy's Prélude à L'Après Midi d'un Faune and Mussorgsky's Night on the Bald Mountain; and Gershwin's Rhapsody in Blue was paired with Glenn Miller's Moonlight Serenade and Gershwin's An American in Paris. Thus, all the alternatives for each choice were plausible, both in terms of composer-title and in terms of melody.

All snippets were recorded onto TDK tapes from original recordings and played to the subjects on a Sony CFS-W301 tape 
recorder. During the recall and FOK phase, the subject's responses were recorded on a sheet of paper. During the recognition test, the subjects were given answer sheets with printed alternatives, to indicate their choices. During the final melody continuation task, the subjects' responses were recorded onto blank tapes, using a General Electric tape recorder with a built-in microphone. A stopwatch was used for all timing purposes.

Design. The 144 snippets were divided into two lists of 72 , one to serve in the name that tune condition, in which the melody was given and the subjects were asked to provide the title, and the other to serve in the hum that tune condition, in which the title was given and the subjects were asked to provide the melody. A total of 24 items made up each list, and the other 48 acted as lure items in the three-alternative forced choice recognition test that followed. The target and the lure items remained as such for all the subjects, although the task order was counterbalanced so that, for half the subjects, List 1 items made up the name that tune condition and List 2 items the hum that tune condition, and, for the other subjects, vice versa. Thus, across subjects, all snippets were tested for their titles and for their melodies equally often. In both recognition tests, the items were presented in a different order from that in the recall and FOK phase.

Each subject went through the recall and FOK task and the recognition test for List 1 and then through the recall and FOK task and the recognition test for List 2. During the recall and FOK phase, the experimenter recorded the subject's response (either an answer or an FOK rating) after each snippet or title. During the recognition test, in one condition, the subjects received a sheet with the titles of the targets printed and indicated which snippet represented each target after listening to the set of three snippets; in the other condition, the subjects received a sheet with three alternative titles printed and circled their choice after listening to each target snippet.

At the end of the second recognition test, the subjects were given all the target snippets they had heard during the experiment and were asked to continue the melody. Finally, the subjects were given a questionnaire about their musical background and music-listening habits.

Procedure. The subjects were tested individually. After the instructions and a few examples, they were given the first recall and FOK rating task. Depending on the condition, they were given a title (or two-for instance, Also Sprach Zarathustra was also identified as the beginning music of the film 2001: A Space Odyssey, and Elgar's Pomp and Circumstance was also identified as the graduation march music) and were asked to hum, whistle, or sing the melody, or they were given a melody and asked to say what its title was. They were given $20 \mathrm{sec}$ to recall the answer. Though they were given instructions not to make wild guesses, they were encouraged to guess, if they could make an educated guess. No feedback was given about wrong answers, and the experimenter went on to the next item after any answer. Thus, following Hart's (1965) original procedure, no FOK ratings were recorded for items for which an answer was given (right or wrong; cf. Krinsky \& Nelson, 1985). If, at the end of the $20 \mathrm{sec}$, they could not recall the answer, the subjects were asked to give an FOK rating of between 1 ( $I$ know for sure I don 't know it) and 6 (I know for sure I know it).

The recognition test for that list followed immediately after the last item. The subjects were given $5 \mathrm{sec}$ to make their decision after hearing all the alternatives for any given title or after hearing any given snippet and looking at all the alternative titles. Then the subjects went on to the second task with the second list (recall and FOK rating and then recognition), using the same procedure.

At the end of the session, after the second recognition test, the subjects listened to another tape, on which the melodies of all 48 target snippets had been recorded. After each snippet, the tape was paused, and the subjects were asked to continue the melody. If they could, their responses were recorded onto a blank tape, to be judged later. It should be pointed out that, in both the recall phase and the FOK phase for the hum that tune condition and in the final continuation phase, an effort was made to create a relaxed atmosphere so that the subjects would not feel inhibited about humming, singing, or whistling; indeed, although there were sometimes apologies about having horrible voices, none of the subjects refused to produce the melody if they thought they knew it. Finally, they filled out a questionnaire about their formal or informal musical training and listening habits.

\section{Results}

Because between-experiment comparisons are risky, there are no statistical comparisons between the results of Experiments 1 and 2. For informal comparison purposes, however, the results of both experiments are summarized in Tables 1-3. Incidentally, contrary to expectations, the subjects classified as musicians (if they had had 5 or more years of training) and listeners (if they listened to more than $20 \mathrm{~h}$ of this type of music every week) did not recall more titles or more melodies than did the other subjects. Thus, because there was no independent verification of our classification of subjects into different categories, we will report only the overall results.

As can be seen in Table 1, the subjects correctly recalled more titles when given melodies as cues than they did melodies when given titles as cues $[t(59)=8.69, p<$ $.01]$. Such an asymmetry in cue efficacy was consistent with intuition. Table 1 also shows the median (and the mean) FOK ratings when recall failed. The subjects gave significantly higher FOK ratings when predicting future recognition of titles than when predicting future recognition of melodies $[t(59)=4.30, p<.01]$. Thus, melody cues evoked better recall of titles, as well as higher FOK ratings, as compared with the recall of melodies and FOK ratings from title cues.

Table 2 shows the results of the recognition test and the subjects' prediction accuracy in terms of gamma measures. An analysis of variance (ANOVA) done on the recognition measures showed a significant main effect for high and low FOK ratings $\left[F(1,59)=114.78, M S_{\mathrm{e}}=\right.$ $290.50, p<.01]$. As can be seen from the table, when the subjects had given an FOK rating from the upper half of the scale, they did, indeed, recognize more titles and melodies than they would have recognized by chance (in title recognition, only 5 subjects did not perform above

Table 1

Percent Recalled Correctly and Median Feeling-of-Knowing (FOK) Ratings (Out of 6) Given No Recall (With Means and $S E M s$ ) When Asked for the Melody With a Title Cue and When Asked for the Title With A Melody Cue

\begin{tabular}{|c|c|c|c|c|c|c|c|c|}
\hline \multirow[b]{3}{*}{ Experiment } & \multicolumn{8}{|c|}{ Asked for } \\
\hline & \multirow[b]{2}{*}{ Melody } & \multirow[b]{2}{*}{ Title } & \multicolumn{3}{|c|}{ Melody } & \multicolumn{3}{|c|}{ Title } \\
\hline & & & Med & $M$ & $S E M$ & Med & $M$ & SEM \\
\hline & \multicolumn{2}{|c|}{ Percent Recalled } & \multicolumn{6}{|c|}{ FOK Ratings } \\
\hline 1 & 8.8 & 18.9 & 2.3 & 3.04 & 0.13 & 3.0 & 3.18 & 0.12 \\
\hline 2 & 21.0 & 17.1 & 4.0 & 3.37 & 0.12 & 3.0 & 3.18 & 0.11 \\
\hline
\end{tabular}


Table 2

Percent Recognized Correctly During the

Recognition Test Phase and Gammas

\begin{tabular}{clcc}
\hline \multirow{2}{*}{ Experiment } & \multicolumn{2}{c}{ Initially Asked for } \\
\cline { 3 - 4 } & & Melody & Title \\
\hline 1 & Overall & 66.2 & 62.0 \\
& High FOK $(4,5,6)$ & 61.0 & 81.4 \\
& Low FOK $(1,2,3)$ & 43.4 & 52.4 \\
\multirow{2}{*}{2} & Gamma & 0.44 & 0.30 \\
& Overall & 65.8 & 67.3 \\
& High FOK $(4,5,6)$ & 71.2 & 71.4 \\
& Low FOK $(1,2,3)$ & 41.3 & 48.5 \\
Note-FOK, feeling of knowing. & 0.47 & 0.38 \\
\hline
\end{tabular}

chance, and, in melody recognition, there was no one who did not) or than they did when they had given a FOK rating from the lower half of the scale $[t \mathrm{~s}(59)=4.52$ and 9.75 for titles and melodies, respectively; both $p s<.01]$. They also recognized titles and melodies above chance level even when they had given low FOK ratings, however $[t \mathrm{~s}(59)=4.49$ and 8.44 , respectively; both $p \mathrm{~s}<.01]$. Interestingly, in contrast with initial recall during the recall and rating phase, in which more titles were recalled when melodies were given as cues than vice versa, during the recognition phase, more melodies were recognized than were titles $\left[F(1,59)=39.95, M S_{\mathrm{e}}=306.75\right.$, $p<.01 ; t(59)=5.75, p<.01$, for items with high FOK ratings, and $t(59)=2.34, p<.03$, for items with low FOK ratings; the interaction between the two conditions was not statistically significant; $F(1,59)=2.41, M S_{\mathrm{e}}=311.48$, $p>.10]$. Of course, because the distractors used in the two recognition tests were different, one needs to be cautious in interpreting this result. That is, although the distractors were of the same pieces, in one case they were made up of titles and in the other melodies, and their recognizability, and thus the likelihood of being ruled out, might have been different.

Gamma measures also showed above-chance accuracy $[t \mathrm{~s}(59)=6.64$ and 11.68 in predicting title and melody recognition, respectively; both $p \mathrm{~s}<.01$ ], and subjects were more accurate in their predictions of whether they could recognize melodies when given their titles than vice versa $[t(59)=2.21, p<.03]$.

Also of interest was the effect of whether subjects could continue a given melody correctly. Overall, $42.7 \%$ of the snippets were continued correctly (we had missing data for the continuation task for one subject, thus his/her responses were excluded from any analyses that involved the continuation task). Continuation data were recorded on tape and later judged to be correct or incorrect by all of the experimenters/authors, depending on whether the second half of the phrase or the next phrase was hummed in its entirety. Without exception, there were no guesses on the subjects' part; if they did not know the melody, they did not attempt a continuation, and if they could continue, there were no ambiguous cases; in fact, we usually had to stop them from going on and on, even after the requisite amount had been hummed.

Table 3 summarizes the results on the basis of whether the melody of each snippet was further recallable or not. Thus, of the melodies that were continued successfully, $17.0 \%$ were recalled during the initial recall and FOK rating phase when subjects were asked for the melody and $41.0 \%$ when they were asked for the title. Of the melodies that could not be continued, however, only $2.2 \%$ were recalled when the subjects were asked for the melody and $4.1 \%$ when they were asked for the title. The $2 \times 2$ ANOVAs on recall, FOK ratings, and recognition all showed statistically significant main effects for further recallability $\left[F(1,58)=199.44, M S_{\mathrm{e}}=197.86\right.$, $F(1,58)=228.06, M S_{\mathrm{e}}=0.607$, and $F(1,58)=79.54$, $M S_{\mathrm{e}}=441.70$, respectively; all $\left.p \mathrm{~s}<.01\right]$. As expected, during the recall and FOK rating phase, significantly more further-recallable melodies (those that were later successfully continued during the continuation task) had been recalled in response to cues, as compared with others $[t(58)=13.90$ when recalling titles and $t(58)=6.90$ when recalling melodies, both $p s<.01]$ and significantly higher FOK ratings had been given to further-recallable melodies than to others $[t(56)=13.69$ when predicting title recognition and $t(58)=8.99$ when predicting melody recognition; both $p s<.01$ ]. Also, during the recognition test, significantly more further-recallable melodies

Table 3

Percent Recalled, Median Feeling of Knowing (FOK) Ratings (Out of 6; With Means and SEMs), Percent Recognized, and Gamma Measures for Melodies for That Were Further Recalled (Continued Correctly) and Melodies That Were Not Further Recalled

\begin{tabular}{|c|c|c|c|c|c|c|c|c|c|c|c|c|}
\hline \multirow[b]{3}{*}{ Condition } & & & \multicolumn{6}{|c|}{ Asked for } & & & & \\
\hline & \multicolumn{2}{|c|}{ Asked for } & \multicolumn{3}{|c|}{ Melody } & \multicolumn{3}{|c|}{ Title } & \multicolumn{4}{|c|}{ Asked for } \\
\hline & Melody & Title & Med & $M$ & $S E M$ & Med & $M$ & $S E M$ & Melody & Title & Melody & Title \\
\hline \multirow{3}{*}{$\begin{array}{l}\text { Experiment } 1 \\
\text { Further recalled } \\
\text { Not further recalled }\end{array}$} & \multicolumn{2}{|c|}{ Recall } & \multicolumn{6}{|c|}{ FOK } & \multicolumn{2}{|c|}{ Recognition } & \multicolumn{2}{|c|}{ Gamma } \\
\hline & 17.0 & 41.0 & 4.0 & 3.79 & 0.14 & 5.0 & 4.32 & 0.14 & 82.9 & 63.7 & 0.43 & 0.03 \\
\hline & 2.2 & 4.1 & 2.0 & 2.44 & 0.12 & 2.0 & 2.60 & 0.11 & 52.9 & 44.7 & 0.40 & 0.23 \\
\hline \multicolumn{13}{|l|}{ Experiment 2} \\
\hline Further recalled & 40.0 & 37.9 & 5.0 & 4.47 & 0.17 & 5.0 & 4.67 & 0.11 & 77.5 & 81.1 & 0.42 & 0.06 \\
\hline Not further recalled & 6.8 & 7.0 & 3.0 & 3.16 & 0.12 & 3.0 & 2.94 & 0.11 & 47.5 & 53.6 & 0.56 & 0.21 \\
\hline
\end{tabular}


had been recognized correctly than had others $[t(58)=$ 4.70 when the task was to recognize the title and $t(58)=$ 9.67 when it was to recognize the melody; both $p s<.01$ ]. Interestingly, as with overall recognition, more melodies were recognized when titles were given as cues than vice versa $\left[F(1,58)=36.13, M S_{\mathrm{e}}=304.10, p<.01\right]$ for both further-recallable and other melodies $[t \mathrm{~s}(58)=5.83$ and 3.14 , respectively; both $p s<.01$ ], but the difference was even more pronounced for further-recallable melodies $\left[F(1,58)=6.45, M S_{\mathrm{e}}=289.09, p<.05\right]$.

With respect to accuracy, however, the subjects were as accurate with further-recallable melodies as with others, both when they predicted the future recognizability of melodies after being given the titles as cues $[t(40)=$ $0.19, p>.10]$ and when they predicted the future recognizability of titles after being given the melodies as cues $[t(39)=1.27, p>.10]$. Separate gamma values for further- recallable and other melodies were incalculable for some subjects because they failed to distribute their ratings sufficiently or had no correct or incorrect responses. Interestingly, when subjects predicted the future recognizability of titles when given the melodies as cues (the condition in which the relationship between further melodic recallability and memory or FOK ratings for verbal titles becomes somewhat more relevant), as mentioned before, they were usually less accurate than when they predicted the future recognizability of melodies $[t(58)=2.21, p<.03]$. In fact, for further-recallable melodies, the subjects' predictions were not even above chance when the task was to predict the future recognizability of titles from the melody cues $[t(40)=0.30, p>.10]$.

In addition, the continuation task could be viewed also as a criterion measure, just like the recognition test, to gauge the accuracy of the FOK ratings. The subjects would be accurate to the extent that they gave high FOK ratings to melodies when presented with titles as cues and indeed could recall the rest of the melody when given the first part or to the extent that they gave low FOK ratings and could not recall the rest of the melody. Such would also be the case when melodies were given as cues, albeit a little less directly for present purposes because the question was whether or not they knew the title. Indeed, the mean gammas were 0.48 after predicting memory for melodies, given the title cues, and 0.70 after predicting memory for titles, given the melody cues; both were above chance level $[t(58)=11.53$ in the former condition, and $t(56)=20.08$ in the latter condition; both $p s<.01]$. The accuracy in predicting memory for melodies, given title cues, was about the same as that obtained when recognition was used as the criterion $[t(57)=0.38, p>.10]$. Unlike the case in which recognition was used as a criterion, however, the subjects were more accurate in predicting memory for the title than in predicting memory for the melody $[t(56)=6.03, p<.01]$. Given that the FOK for the title was probed with the first part of the melody, it appears that, consistent with intuition, the subjects thought about the rest of the melody and based their predictions of memory for the title on whether they knew the mel- ody. And because this criterion measure was asking for the rest of the melody, the subjects were indeed quite accurate, much more so than when recognition of the title was the criterion measure, as was promised $[t(56)=$ $7.64, p<.01]$.

\section{EXPERIMENT 2}

In this experiment, the snippets of melodies that were used were from songs with lyrics. Again, they were selected through extensive piloting, as described in Experiment 1 , and some examples include the theme song of the television show "The Brady Bunch," Love Story, Blowing in the Wind, Sunrise, Sunset, Misty, and Sound of Silence. The snippet was always from the beginning of the song (where the lyrics started) and lasted as long as the lyrical phrase did, even though the lyrics were not presented, and the song was unambiguously identifiable; the mean length was $4.9 \mathrm{sec}$ with a range of $2.1-8.5 \mathrm{sec}$. In a study by Hyman and Rubin (1990), it was found that the greatest predictor of the probability of remembering a title was when a cue line of lyrics from the song shared words with the title. In the present case, the snippets we picked never contained the title as part of the lyrics, even though, again, the lyrics were not presented either. Thus, the melodies were usually not the best-known parts of the songs, as might have been their refrain sections that contained the title as part of the lyrics. In this way, we hoped we would maximize our chances of obtaining FOK states, rather than getting immediate recall. Unlike in the previous experiment, the snippets were not from the original recordings but were recorded onto the tapes from a Kawai FS 630 keyboard, using the soft piano setting. Thus, there were no cues, such as the singer's voice or orchestration, other than the simple melody the nonexistent lyrics followed. The method was identical to that of the previous experiment, and the subjects were 60 students at American University, who received extra credit toward psychology courses. According to questionnaire results, 20 had had 5 or more years of musical training and 21 listened to $20 \mathrm{~h}$ or more of music each week ( 7 belonged to both categories). But because we again failed to get independent evidence for classifying the subjects into these groups of expertise, all the data were collapsed.

\section{Results}

The results are summarized in Tables 1-3 along with those of Experiment 1 . As can be seen in Table 1, during the recall and FOK rating phase of the experiment, the subjects correctly recalled more melodies when given the titles as cues than they did titles when given the melodies as cues $[t(59)=2.09, p<.05]$. Interestingly, however, this asymmetry was in the opposite direction from that observed in Experiment 1. In Experiment 1, recall was better for titles than for melodies, whereas here recall was better for melodies than for titles. Also in Table 1 are the median (and the mean) FOK ratings when recall failed. The subjects gave higher ratings when pre- 
dicting future recognition of melodies as opposed to titles, though, statistically, this trend was only marginally significant $[t(59)=1.50, p<.10]$. It appeared that, again in contrast with Experiment 1, title cues tended to evoke higher FOK ratings for the melody than vice versa.

A $2 \times 2$ ANOVA revealed a statistically significant main effect of FOK ratings in the recognition tests $[F(1,59)$ $\left.=183.47, M S_{\mathrm{e}}=227.96, p<.01\right]$. As can be seen in Table 2 , when subjects had given a FOK rating from the upper half of the scale, they recognized more titles and melodies than they would by chance (only 1 subject failed to do so in each condition) or than they did when they had given a FOK rating from the lower half of the scale $[\operatorname{ts}(59)=7.90$ and 9.66 for titles and melodies, respectively; both $p s<.01]$. As in Experiment 1, however, the subjects also recognized titles and melodies above chance level, even when they had given low FOK ratings $[t \mathrm{~s}(59)=$ 7.01 and 3.16, respectively; $p s<.01]$. Although more melodies were recalled than were titles during the recall and FOK rating phase, during the test phase the percentages of correctly recognized melodies and titles were about the same $\left[F(1,59)=3.32, M S_{\mathrm{e}}=246.63, p>.05\right]$; the only difference that emerged was for items with low FOK ratings, in which case more titles were recognized correctly than were melodies $[t(59)=2.43, p<.02]$. Again, because the two tests used different distractors (melodies and titles), one needs to be cautious in interpreting this result.

Gamma measures also showed above-chance accuracy $[t \mathrm{~s}(59)=9.67$ and 12.36 for predicting title and melody recognition, respectively; both $p \mathrm{~s}<.01$ ] and, similar to the results of Experiment 1, the subjects were marginally more accurate in their predictions of whether they could recognize melodies when given their titles than vice versa $[t(59)=1.69, p<.09]$.

In the further melodic recall task, overall $39.7 \%$ of the snippets were continued correctly. Again, $2 \times 2$ ANOVAs on recall, FOK ratings, and recognition all showed statistically significant main effects of further recallability $\left[F(1,59)=218.87, M S_{\mathrm{e}}=341.63 ; F(1,59)=155.96\right.$, $M S_{\mathrm{e}}=0.889$; and $F(1,59)=115.50, M S_{\mathrm{e}}=429.00$, respectively, all $p \mathrm{~s}<.01]$. As can be seen in Table 3, more further-recallable items were recalled during the recall and FOK rating phase than were others $[t(59)=10.50$, when asked for the melody, and $t(59)=8.28$, when asked for the title; both $p s<.01]$. Also, higher FOK ratings were given to further-recallable melodies than to others $[t(56)=13.39$, when asked for the melody, and $t(58)=$ 7.34, when asked for the title; both $p$ s $<.01]$.

During the recognition test, more further-recallable melodies were recognized correctly than were others $[t(59)=8.77$, when the task was to recognize the melody, and $t(59)=7.50$, when it was to recognize the title; both $p s<.01]$. Interestingly, unlike what was found in Experiment 1 , regardless of whether the melodies were further recallable or not, melodies were not recognized more often than were titles; in fact, this time titles were recognized more often $\left[F(1,59)=4.14, M S_{\mathrm{e}}=334.35, p<.05\right]$.
With respect to accuracy, the results were quite similar to those of Experiment 1. Prediction accuracy of future recognizability for both melodies and titles was unaffected by further melodic recallability $[t \mathrm{~s}(32)$ and $(27)=0.68$ and 0.98 , respectively, both $p \mathrm{~s}>.10]$. The subjects were less accurate in their predictions of future recognizability of titles (the condition in which the relationship between further melody recallability and memory or FOK ratings for titles is more interesting) than in those of melodies, albeit only marginally so this time $[t(59)=1.69, p<.10]$; strikingly, this was true mainly for melodies that could not be continued $[t(58)=3.80$, $p<.01]$ rather than for those that could be $[t(21)=0.86$, $p>.10]$. Again, the subjects' predictions were not even above chance level for further-recallable melodies when the task was to predict future recognizability of titles from the melody cues $[t(30)=0.17, p>.10]$.

When the continuation task was treated as a criterion measure, the gammas were 0.50 after predicting memory for melodies given the title cues and 0.67 after predicting memory for titles given the melody cues; both were above chance level $[t \mathrm{~s}(57)=8.84$ and 14.32 , respectively; both $p s<.01]$. As in Experiment 1, the accuracy in predicting memory for melodies, given title cues, was not different from that when recognition was used as the criterion $[t(57)=0.55, p>.10]$, and, with the continuation task as the criterion, the subjects were more accurate in predicting memory for titles than for melodies $[t(55)=$ $5.11, p<.01]$. In addition, again as in Experiment 1 and in line with intuition, the subjects were more accurate in predicting memory for the titles when the continuation task was used as the criterion than when recognition was $[t(57)=7.58, p<.01]$.

\section{CONCLUSIONS}

We explored memory for melodies and titles of music, as well as FOKs when recall failed. With instrumental music, people recalled titles (verbal targets) when given melodies as cues more often than they recalled melodies (nonverbal auditory targets) when given titles as cues. This asymmetry reversed itself with music that had lyrics. Although the lyrics that went with the melodies were absent, melodies were recalled more often than the titles. Thus, consistent with research on the integration of melodies and words in songs (e.g., Serafine et. al., 1984; Serafine et. al., 1986), words in songs may have a mediating effect between memory for the title and memory for the melody. Although the nature of such mediation is far from clear, the verbal title may cue the verbal text, which can then cue the melody, which is more effective than the melody cuing the text and the text then cuing the title.

Of course, because the presence or absence of lyrics was a between-experiment manipulation, we cannot compare the two sets of results. There were also other differences in the two types of music. For instance, the titles of the second set of pieces tended to be distinctive more often than were those of the first set (e.g., Pachelbel's Canon vs. 
Blowing in the Wind) and perhaps such titles were also more representative of the melody. Thus, at this stage, any comparisons between memory or metamemory for music with and without lyrics are merely speculative. But, at the very least, the findings within each experiment suggest that the effectiveness of various cuing strategies is not symmetrical. It appears that cuing a melody with its title and cuing the title with the melody may lead to vastly different results in eliciting memory.

We also found that FOK ratings for both melodies and titles were above-chance predictors of future remembering. Thus, it appears that, just as with verbal materials, people are able to make somewhat accurate FOK judgments about musical materials, too. This was the case when a recognition test was used as the criterion measure as well as when a further melodic recall task was. Consistent with their performance in actual recall, subjects gave higher FOK ratings in predicting future remembering of titles as opposed to melodies with instrumental music, and vice versa for music lyrics. These ratings were more accurate predictors of future remembering of melodies than of future remembering of titles in both types of music, however.

Finally, we found that, as expected, melodies that subjects were able to continue later on had been more often recalled or given higher FOK ratings during the initial recall and FOK rating phase, as well as better recognized during the recognition phase, both when given the title as a cue and asked for the melody and vice versa. Such was the case in both experiments. Perhaps more interestingly, although higher FOK ratings had been given to melodies that subjects were later able to recall further and continue, these ratings were not accurate at all. The subjects were more accurate in their FOK ratings for melodies that they could not continue. In fact, when the cue was the melody and the task was to predict the future recognizability of its title, prediction accuracy did not exceed chance level for further-recallable melodies in either experiment. Thus, it appeared that the subjects' FOK ratings for predicting future recognizability of titles from melody cues was, at least in part, based on whether they could recall the melodies further (as was also evidenced by the much greater accuracy subjects showed when the criterion measure was to actually continue the melody). Consistent with the idea that melodies and their titles are often unrelated, however, using such a strategy turned out to work against the subjects, because the accuracy of these ratings suffered considerably.

\section{REFERENCES}

Bartlett, J. C., \& SNelus, P. (1980). Lifespan memory for popular songs. American Journal of Psychology, 93, 551-560.

GruneberG, M. M., \& Monks, J. (1974). Feeling of knowing and cued recall. Acta Psychologica, 38, 257-265.

HART, J. T. (1965). Memory and the feeling-of-knowing experience. Journal of Educational Psychology, 56, 208-216.

Hyman, I. R., JR., \& Rubin, D. C. (1990). Memorabeatlia: A naturalistic study of long-term memory. Memory \& Cognition, 18, 205-214.

KRINSKY, R., \& NELSON, T. O. (1985). The feeling of knowing for different types of retrieval failure. Acta Psychologica, 58, 141-158.

Nelson, T. O., Gerler, D., \& Narens, L. (1984). Accuracy of feelingof-knowing judgments for predicting perceptual identification and relearning. Journal of Experimental Psychology: General, 113, 282300.

Nelson, T. O., McSpadden, M., Fromme, K., \& Marlatt, G. A. (1986). Effects of alcohol intoxication on metamemory and on retrieval from long-term memory. Journal of Experimental Psychology: General, 115, 247-254.

SCHACTER, D. L. (1983). Feeling of knowing in episodic memory. Journal of Experimental Psychology: Learning, Memory, \& Cognition, 9, 39-54.

Serafine, M. L., Crowder, R. G., \& Repr, B. H. (1984). Integration of melody and text in memory for songs. Cognition, 16, 285-303.

Serafine, M. L., Davidson, J., Crowder, R. G., \& Repp, B. H. (1986). On the nature of melody-text integration in memory for songs. Journal of Memory \& Language, 25, 123-135.

\section{NOTES}

1. Although the issue of meaning in music is still open to discussion, we would like to avoid this debate for present purposes because, whatever the position we take (e.g., that of a referentialist or an absolutist), it is still the case that titles of melodies often have little to do with the meaning conveyed by those melodies.

2. A list of all items for both experiments is available, on request, from the first author.

(Manuscript received April 12, 1995 ; revision accepted for publication July 28,1997 .) 